OR-20

\title{
SYNTHESIS AND STRUCTURE-ACTIVITY RELATIONSHIPS OF THE NOVEL ISOTHIOBARBAMINE ANALOGUES WITH LOWERED BASICITY
}

\author{
A. Novakov, ${ }^{1}$ L. L. Brunilina, ${ }^{1}$ V. V. Chapurkin, ${ }^{1}$ M. B. Nawrozkij,${ }^{1}$ D. S. Sheikin ${ }^{1}$ \\ Y. P. Kuznetsov, ${ }^{2}$ E. A. Ruchko, ${ }^{3}$ D. Schols ${ }^{4}$ \\ ${ }^{1}$ Volgograd State Technical University, Lenin Ave., 28, Volgograd, 400005, Russia; \\ ${ }^{2}$ Volzhsky Polytechnic Institute (Branch), Volgograd State Technical University, 42 Engelsa St., \\ Volzhskii, Volgograd obl., 404121, Russia. \\ 3 "'Pharm-Sintez" Closed Corporation, 2-ya Kabel'naya St., 2, bld. 46, Moscow, 111024 Russia; \\ ${ }^{4}$ Rega Institute for Medical Research, KU Leuven, Laboratory of Virology and Chemotherapy, \\ Minderbroedersstraat 10, B-3000, Leuven, Belgium \\ E-mail: d.sheikin@yandex.ru
}

Isothiobarbamine is a synthetic adaptogen, derived from 6-hydroxy-5-isopropyl-2-thiouracil:<smiles>CCCCCSc1nc(O)c(C(C)C)c(=O)[nH]1</smiles>

It was first described by Ukrainian scientists and showed a remarkable profile of biological activity in different in vivo tests, combined with low acute toxicity. Taking into consideration the results of L. Monti and G. Franchi on the structure-activity relationships of 5-(aminomethyl)-2-thiouracil derivatives versus the corresponding 5-(amidomethyl)-counterparts, we designed and prepared a series of different Isothiobarbamine analogues with lowered basicity, carrying an amido-function instead of diethylaminogroup, together with various substituents of the pyrimidin-4(3H)-one fragment:

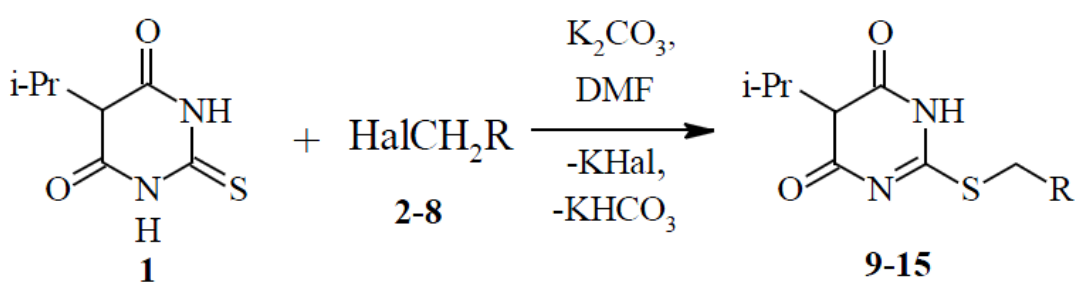

$\mathrm{R}=\operatorname{PhthN}(\mathbf{2 , 9}), \mathrm{PhthNCH}_{2}(\mathbf{3 , 1 0}), \mathrm{PhthN}\left(\mathrm{CH}_{2}\right)_{2}(\mathbf{4 , 1 1})$, Hal = Br.

$\mathrm{R}=\mathrm{C}(\mathrm{O}) \mathrm{NEt}_{2}(\mathbf{5 , 1 2}), \mathrm{C}(\mathrm{O}) \mathrm{N}\left(\mathrm{CH}_{2}\right)_{4}(\mathbf{6 , 1 3}), \mathrm{C}(\mathrm{O}) \mathrm{N}\left(\mathrm{CH}_{2}\right)_{5}(\mathbf{7 , 1 4}), \mathrm{C}(\mathrm{O}) \mathrm{N}\left(\mathrm{CH}_{2} \mathrm{CH}_{2}\right)_{2} \mathrm{O}(\mathbf{8}, \mathbf{1 5}), \mathrm{Hal}=\mathrm{Cl}$.

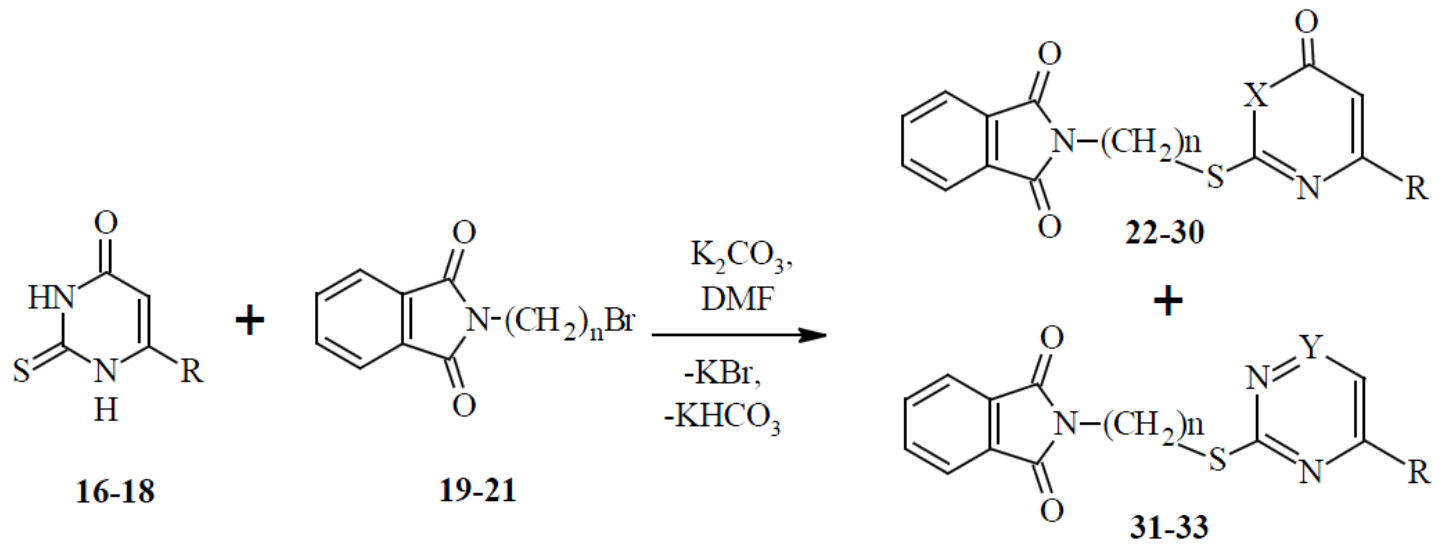

Где: $\mathrm{R}=\mathrm{NH}_{2}, \mathrm{n}=2(\mathbf{2 2}), 3(\mathbf{2 3}), \mathrm{X}=\mathrm{NH}$;

$\mathrm{R}=\mathrm{Me}, \mathrm{n}=1(\mathbf{2 4}), 2(\mathbf{2 5}), 3(\mathbf{2 6}), \mathrm{X}=\mathrm{NH} ; \mathrm{n}=2(\mathbf{3 1}), 3(\mathbf{3 2}), \mathrm{Y}=\mathrm{CO}\left(\mathrm{CH}_{2}\right)_{\mathrm{n}} \mathrm{NPhth}$;

$\mathrm{R}=\mathrm{CF}_{3}, \mathrm{n}=1(\mathbf{2 7}), 2(\mathbf{2 8}), 3(\mathbf{2 9}), \mathrm{X}=\mathrm{NH} ; \mathrm{n}=2(\mathbf{3 0}), \mathrm{X}=\mathrm{N}\left(\mathrm{CH}_{2}\right)_{2} \mathrm{NPhth} ; \mathrm{n}=2(\mathbf{3 3}), \mathrm{Y}=\mathrm{CO}\left(\mathrm{CH}_{2}\right)_{2} \mathrm{NPhth}_{\text {. }}$

The latter derivatives showed some anti-HIV-1/2 activity, together with low toxicity in cellular assays. 